\title{
Study of Magnetic Interference on an LVDT: FEM Modeling and Experimental Measurements
}

\author{
Alessandro Masi, ${ }^{1}$ Alessandro Danisi, ${ }^{1,2}$ Roberto Losito, ${ }^{1}$ \\ Michele Martino, ${ }^{1,3}$ and Giovanni Spiezia ${ }^{1}$ \\ ${ }^{1}$ EN-STI Group, European Organization for Nuclear Research (CERN), CH-1211 Geneva 23, Switzerland
${ }^{2}$ IMT-LAI Laboratory, École Polytechnique Fédérale de Lausanne (EPFL), CH-2002 Neuchâel, Switzerland
${ }^{3}$ Electrical Engineering Department, University of Naples "Federico II", Via Claudio, 21-80125 Naples, Italy
}

Correspondence should be addressed to Alessandro Danisi, alessandro.danisi@cern.ch

Received 30 September 2010; Revised 7 February 2011; Accepted 18 March 2011

Academic Editor: Andrea Cusano

Copyright (c) 2011 Alessandro Masi et al. This is an open access article distributed under the Creative Commons Attribution License, which permits unrestricted use, distribution, and reproduction in any medium, provided the original work is properly cited.

\begin{abstract}
Static or slowly varying magnetic fields can affect the performances of linear variable differential transformer by inducing a position reading drift. The problem is barely addressed in LVDTs' datasheets, and no quantitative information on the induced error is given. An LVDT finite element model is here presented together with its experimental validation in order to propose a tool for the study of the effects of external magnetic fields on LVDTs and for the design of less sensitive devices. The LVDT model has been validated in standard working conditions and in presence of an external magnetic field by means of a complete set of experimental measurements performed on a custom prototype, manufactured following the FEM guidelines.
\end{abstract}

\section{Introduction}

The linear variable differential transformer is a magnetic position transducer. Its use is very widespread due to its features of being a contactless sensor, with virtually infinite resolution and high accuracy [1]. Resolution and accuracy are mainly determined by the conditioning electronics [2-4] and can be improved by means of ratiometric reading [5]. Its use is, therefore, quite common in harsh environments such as nuclear plants and particle accelerators [6].

The LVDT sensor is basically a transformer with one primary winding, in the center of the cylindrical structure, and two secondary windings, one of each side of the primary, wound on a cylindrical support (Figure 1). A ferromagnetic core can move along the axis, and the flux linkage between the primary and secondary windings changes accordingly to its position. In particular, when the core is completely on one side of the structure, one secondary voltage is maximum whereas the other one is minimum. When the core is in the middle, the two voltages are equal. The position of the core can be extracted by a differential reading of the secondary signals. The frequency used for the feeding signal is often in the range of a few $\mathrm{kHz}[1]$. Usually, the core is made up of Ni-Fe alloy, a material which exhibits high magnetic permeability and commonly used for these applications $[7$, 8].

Although the LVDT reading accuracy can be guaranteed even in critical and noisy installations [6], this sensor has shown to be sensitive to external slowly varying magnetic fields [9]. This is the case, for example, of installations next to devices with significant leakage or generated fields, such as motors or high current cables. The influence of such devices can lead to a position reading error which can be of the order of magnitude of hundreds of micrometers. It is, therefore, evident that this kind of error can represent a serious issue in applications where nominal accuracy of a few microns is required [6]. To the authors' knowledge, this problem is barely addressed in a few LVDT data sheets and completely overlooked by the vast majority of the producers; even when a warning is present, no quantitative indications on the induced errors on the position reading, nor about possible countermeasures, are present. In the scientific literature, this problem has been firstly introduced and discussed by [912], although no quantitative validated models are available. 


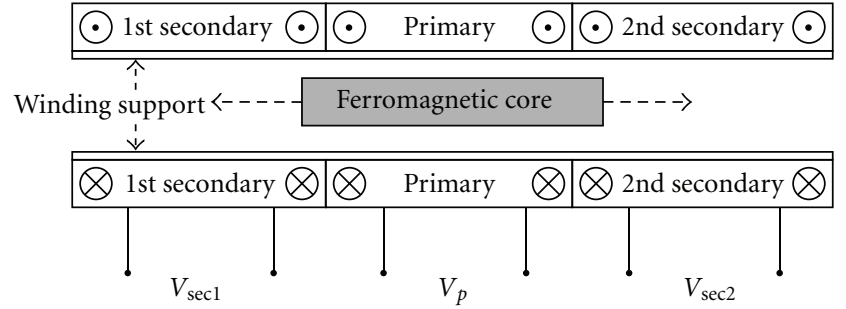

FIGURE 1: Typical LVDT layout and working principle (longitudinal section).

In this paper, a finite element (FE) model of linear variable differential transformer for the magnetic interference study is presented, in order to conceive a tool to (i) study and quantify the interference effects, (ii) aid the conception and the development of an analytical model of the phenomenon, and (iii) design possible countermeasures for existing LVDTs or completely immune structures.

FEM-aided analysis allows a deeper investigation regarding local variables and physical magnitudes, such as local values of magnetic flux density or magnetic field $[13,14]$, practically very difficult or too expensive to measure directly.

The results coming from the FE analysis are then validated on a customized LVDT prototype, whose structure closely reflects the one of the finite elements model itself. A complete set of measurements has been performed on the manufactured prototype, in standard working conditions and in presence of an external interfering magnetic field, in order to verify the agreement with the model. Since the LVDT can be supplied by either voltage $[4,15]$ or current excitation [16], the measurements have been performed in both cases, in order to point out possible differences and eventually compare the interference effects in different supply cases.

In Section 2, the finite element model and the simulation outline are presented. The simulation results are discussed in Section 3. In Section 4, the measurements setup and the experimental results are presented and the comparison with the simulations is then discussed.

\section{LVDT Finite Element Model and Simulation Procedure}

An FEM model for the simulation of the LVDT sensor has been developed using the simulation software FLUX. This simulator is particularly suited for the finite element analysis of electromagnetic problems involving 2D and 3D geometries [17].

The purpose of such an FEM analysis is to conceive a model of linear variable differential transformer which can be used as a tool for analysis and design of LVDT exposed to external magnetic fields. The availability of such a model would allow an immediate feedback in the analytical study of the physical phenomenon of the external magnetic fields influence on LVDT reading, as well as in the design process of an LVDT-like structure with immunity to external magnetic fields.
TABLE 1: Mesh and solving information.

\begin{tabular}{lc}
\hline Info & Parameters \\
\hline Mesh type & 2D triangular mesh \\
$\begin{array}{l}\text { Mesh order and density } \\
\text { General meshing rule }\end{array}$ & 2nd order, nonuniform \\
Number of poor elements & $3-4$ elements per thickness \\
$\begin{array}{l}\text { Number of nodes } \\
\text { Solving scenario }\end{array}$ & $2.8 \%$ \\
$\begin{array}{l}\text { Target relative epsilon of } \\
\text { the Newton-Raphson solver }\end{array}$ & About 43000 \\
$\begin{array}{l}\text { Maximum number of iterations } \\
\text { Solving time (simple computation: } \\
\text { no parameters except time) }\end{array}$ & Time transient \\
\hline
\end{tabular}

Being in principle the external field not known in many applications (neither in terms of amplitude, nor in terms of time evolution), a recalibration of the device in presence of interfering field, which could be an effective solution for purely DC fields, cannot be of help in this case. Furthermore, the external field may not be always present. Indeed, in presence of a slowly varying external field (i.e., the frequency content of such a field being in the ultralow frequency range whereas the internal LVDT magnetic field harmonics are in the range of few $\mathrm{kHz}$ ), at each reading the LVDT will experience an error which is a function of the value of the interfering field (which is nearly constant). Therefore, at each reading, the effect on the position can be seen as due to a DC external field. However, the existence of an intrinsic time variation of such a field, for what has been said, invalidates the possibility to recalibrate the device.

2.1. 2D Modeling. The LVDT geometry presents cylindrical symmetry. On the other hand, an interfering magnetic field impinging the LVDT structure can be in principle arbitrarily oriented. However, an arbitrarily oriented magnetic field can be seen as the superposition of longitudinal (parallel to the LVDT axis) and transversal (perpendicular to the axis) components. Given that such sensor is more sensitive to longitudinal magnetic fields [10], this case is here considered. Thus, the simulation geometry has to include the sensor itself and an external longitudinal magnetic field source. Actually, the impinging magnetic field (the magnetic field generated by the external source when the LVDT is not present) can be uniform or nonuniform along the LVDT axis. For a firststep model, the interfering magnetic field has been chosen to be uniformly distributed along the axis and on the crosssection. For this reason, the interfering magnetic field will exhibit a rotational symmetry too. Thus, the magnetic field source can be a solenoid. In this way, the whole structure has complete cylindrical symmetry and the simulation geometry can be built in two dimensions.

The simulated LVDT model slightly differs from the simple structure presented in Section 1 and displayed in Figure 1. In the actual model, the primary coil is indeed wound on the entire length of the winding support whereas the secondary coils are wound over the primary, one of each 


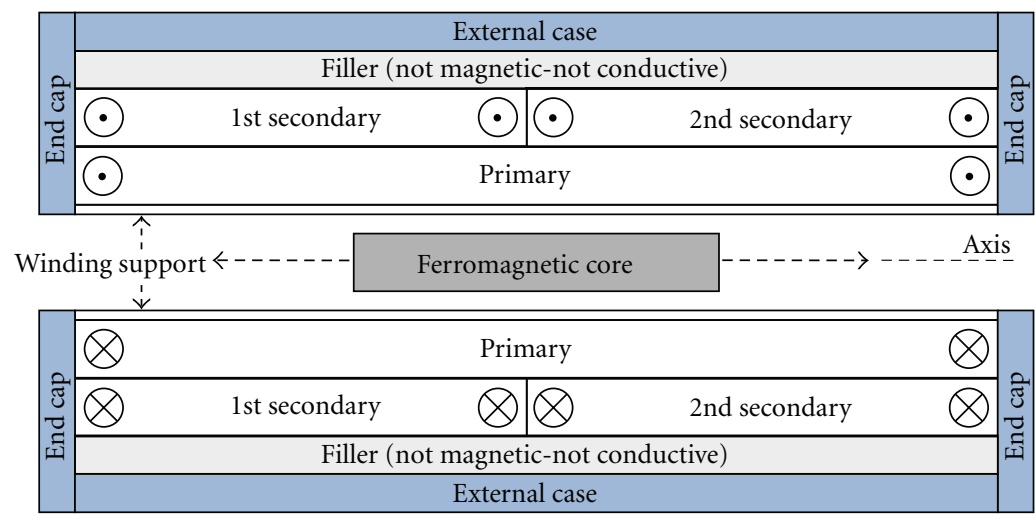

FIGURE 2: 2D longitudinal scheme of the LVDT sensor FEM model (not in scale).

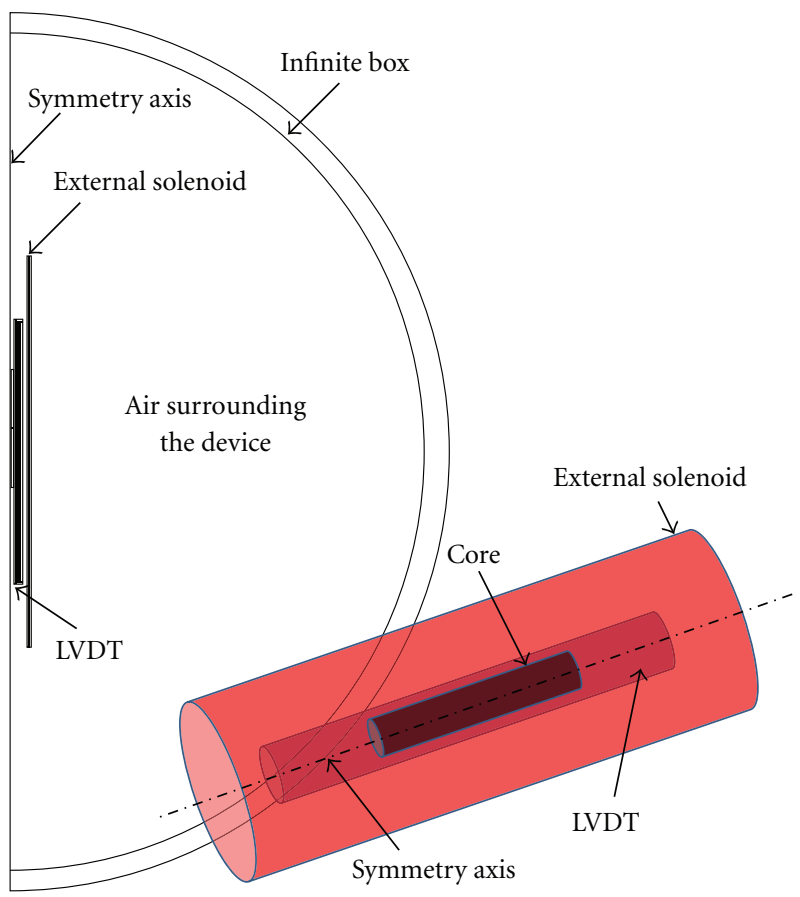

Figure 3: 2D simulation geometry and 3D reconstruction. In the infinite box, a geometrical transformation is performed in order to simulate the infinite space [13].

side of the structure (Figure 2). In this way, the leakage inductances of the transformer are significantly reduced. The model presents insulator washers and layers, treated as nonmagnetic regions.

The structure is enclosed in a ferromagnetic cylindrical case with two end caps. The ferromagnetic case, together with the end caps, has two main functions: it closes the LVDT magnetic circuit and acts like a first shielding against external magnetic fields. The core is a cylinder whose length is equal to the secondary coils length.

Given the cylindrical symmetry, the simulation geometry takes into account only half of the longitudinal section of the sensor (Figure 3). Actually, the complete 3D geometry is obtained by rotating the simulation geometry around the symmetry axis by 360 degrees (Figure 3 ). However, even though the finite element analysis can be performed totally in the $2 \mathrm{D}$ environment, the results are provided anyway for the whole volume of the device, resulting in a significant reduction of computational time.

The structure has a high aspect ratio, thus a fine mesh has been chosen in order to discretize the thicknesses whereas the mesh along the length of the sensor can be coarser. By doing so, the mesh has been optimized using triangular elements on all the geometry. The meshing and the solving parameters for the geometry are reported in Table 1 . The presence of a small amount of poor elements (i.e., nearly flat triangular elements), disposed axially, is not a concern; indeed in such a structure, the variation of the fields in a single region is supposed to be more rapid in the transversal direction, rather than in the longitudinal one [18]. This assumption applies also to the regions of the structure between the coils and the external case, in which the poor elements are present. In addition, in the regions corresponding to the magnetic media, the mesh density has been adapted to the penetration depth. Being in principle the magnetic permeability a function of the magnetic field, the penetration depth has been calculated in the worst case (i.e., maximum permeability) and the meshing density arranged so as to have at least two meshing elements inside the skin depth area [17].

2.2. Magnetic Materials. The FEM modeling of magnetic materials properties for the study of the LVDT magnetic interference is not trivial. As a matter of fact, given the presence of an external magnetic field superposed to the one due to the LVDT working principle, the materials magnetic properties have to be correctly described in all regions of the $\mathrm{BH}$ plane for the simulations to closely match their actual behavior. Unfortunately, FEM simulators model the nonlinearity of magnetic materials by considering their normal magnetization curve, without taking into account major and minor hysteresis effects [17].

For these reasons, the materials which have been chosen for the magnetic parts of the LVDT sensor model had been through an annealing procedure. The preference of having annealed samples instead of not treated materials lies in the fact that in annealed state such samples exhibit high 
permeability and narrow hysteresis cycle [19], as well as a uniformly distributed magnetic permeability [1]. These properties make them more suitable to be modeled with a finite element approach.

PERMENORM $5000 \mathrm{H} 2$ (50\% Ni-Fe alloy) has been chosen as core material whereas ferromagnetic steel has been used for the cylindrical case, since these materials were available to be used and they had been through an annealing procedure.

Regarding the coils' specifications, a number of turns of 1500 has been chosen for the primary winding, in order to have a sufficiently high field inside the sensor whereas a number of turns of 1600 has been chosen for both secondary coils, setting the transformation ratio.

2.3. Solving Strategy. The time transient solver has been chosen. The Newton-Raphson method is used for nonlinear solving and a maximum number of 100 iterations is set for each time instant. The relaxation factors for this algorithm are calculated through the Fujiwara method [17].

Geometrical distances, including crucial parameters (e.g., the core displacement) have been parameterized in order to allow rapid parametric simulations embedded in the same simulation scenario. The numerical transients have been avoided by using an initialization by static computation [17]. On the other hand, for each simulation, an adequate time window has been chosen for the time transient computation, in order to avoid physical transient phenomena.

The parameterization of the core displacement allows performing a set of simulations at different core positions. For each of them, the geometry is remeshed and the time transient computation performed [17]. The core position range which has been set goes from $-20 \mathrm{~mm}$ to $20 \mathrm{~mm}$ in 11 steps with constant spacing. By doing so, the simulation in standard working conditions results in the sensor's calibration.

For the interfering field simulation, taking critical installations as an example $[6,9]$, the case of a $1 \mathrm{mT}$ external magnetic flux density has been considered, which in air corresponds to an interfering magnetic field of about $800 \mathrm{~A} / \mathrm{m}$. The magnetic field is axially oriented and spatially uniform.

\section{Simulation Results}

The simulation results for voltage and current supply, in presence and in absence of the interfering magnetic field, are here shown.

Although the two supply modes are both used in commercial LVDTs, the need of studying the phenomenon with two different supplies comes from the fact that when the supply signal is a purely sinusoidal current, the overall magnetic field is sinusoidal whereas with voltage supply the overall magnetic field is distorted due to the nonlinearity of the magnetic media. Hence, the influence of the external magnetic field can, in principle, play different roles in the two examined cases.
For each supply case, the LVDT characteristic curve, which shows the first harmonics of the secondary voltages with respect to the reference position, is shown. In addition, the ratiometric has been computed as follows:

$$
r=\frac{V_{1}-V_{2}}{V_{1}+V_{2}}
$$

where $V_{1}$ and $V_{2}$ are the first harmonics of first and second secondary, respectively. The relative ratiometric-position curves are displayed. On these curves, a nonlinearity error has been computed as

$$
\mathrm{NLE}=100 \cdot \frac{\max \left(p^{\prime}-p^{*}\right)}{\mathrm{CPR}},
$$

where $p^{*}$ is the core reference position, $p^{\prime}$ is the position calculated through linear interpolation of the relationship between ratiometric and reference position, and CPR is the core position range. Even if normally the core position range goes from $-20 \mathrm{~mm}$ to $20 \mathrm{~mm}$, some interesting results hold for smaller ranges too.

In case of interference, the external magnetic field yields an error on the measured position. Hence, a position drift has been computed, at each core position, as the difference between the position read in presence of the external interference and the position read in absence of the interference. In both cases, the position is read using the ratiometric reading technique, by means of the calibration curve obtained in standard working conditions. Actually the position drift allows performing a relative study (i.e., a study on a position variation) in order to highlight the effect of the external magnetic field on a certain position.

3.1. Voltage Supply. The sensor has been supplied by a $3.5 \mathrm{~V}$ peak sinusoidal voltage at $2000 \mathrm{~Hz}$. The characteristic curve in this case is displayed in Figure 4 (dotted line) for core positions going from $-20 \mathrm{~mm}$ to $+20 \mathrm{~mm}$.

It can be noticed that the two curves have a dual behavior in terms of trend, which recalls the working principle of LVDT sensors. The curve is symmetric with respect to the null position, due to the complete symmetry of the device. The simulations show that the secondary transformation ratio of the sensor goes from 0.54 (minimum core coupling) to 1.66 (maximum core coupling).

The behavior of the ratiometric as a function of the position has been evaluated, and the relative results are depicted in Figure 5 (dotted line). The ratiometric is a monotonic function rising from about -0.5 to about 0.5 over the full core position range. Once again, the perfect odd symmetry of the curve reflects the perfect geometrical symmetry and reciprocity of the simulated device.

In simulation, the nonlinearity of the device resulted, $0.7 \%$ in the range $(-20 \mathrm{~mm}, 20 \mathrm{~mm}$ ). The linearity becomes more acceptable when the core position range is reduced: nonlinearity error is $0.11 \%$ in the range $[-10 \mathrm{~mm},+10 \mathrm{~mm}]$.

The results for the longitudinal interference case are displayed in Figure 8 (dotted line). Some interesting remarks can be pointed out by looking at this graph. The position drift is a monotonic function of the core position and 


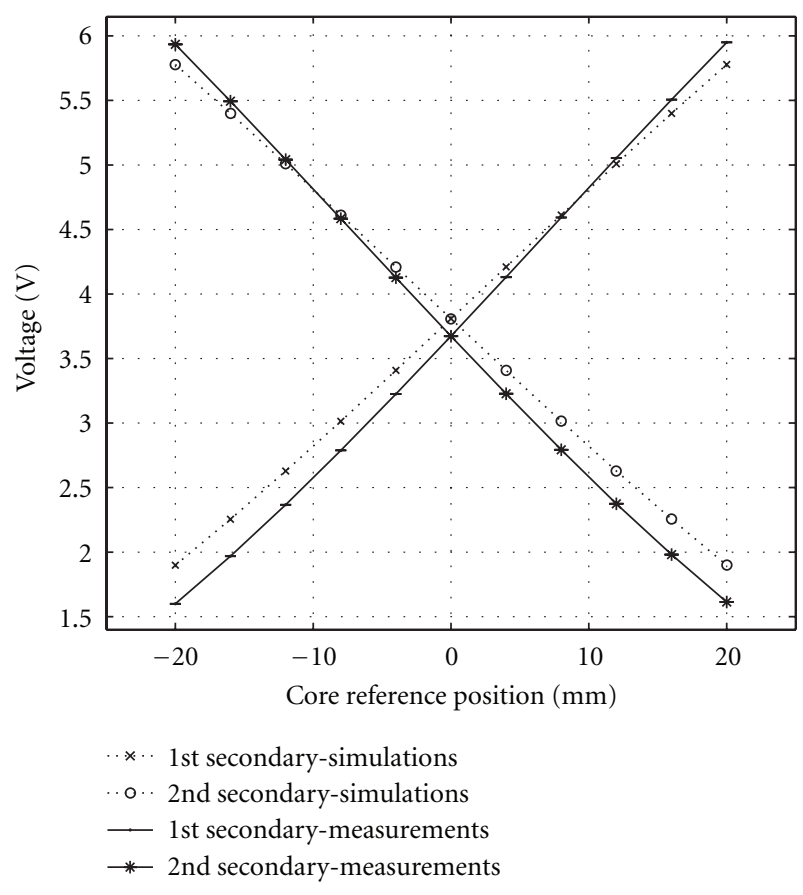

FIGURE 4: Simulations and measurements results regarding the LVDT secondary characteristic curve (voltage supply). The measurement data are depicted with the corresponding uncertainty.

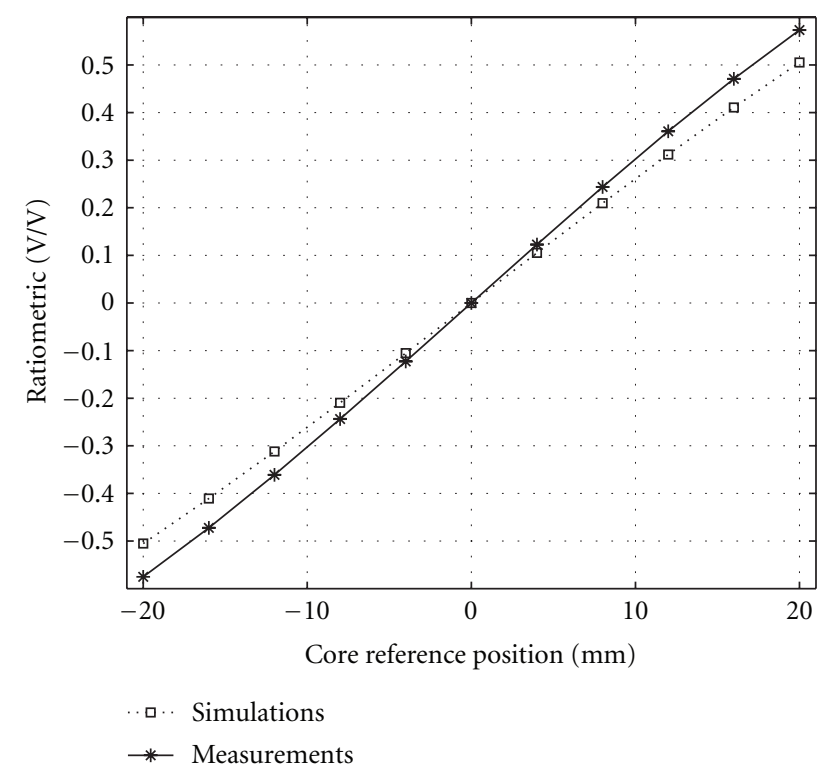

FIGURE 5: Simulations and measurements results regarding the ratiometric (voltage supply). The measurement data are depicted with the corresponding uncertainty.

it exhibits an odd symmetry (which again reflects the geometrical symmetry of the device). In addition, it is zero in the null position. The farther from the center the core is, the greater the position error is. In the worst case, in which the core position is $20 \mathrm{~mm}$, the position drift can reach a value of about 70 micrometers.

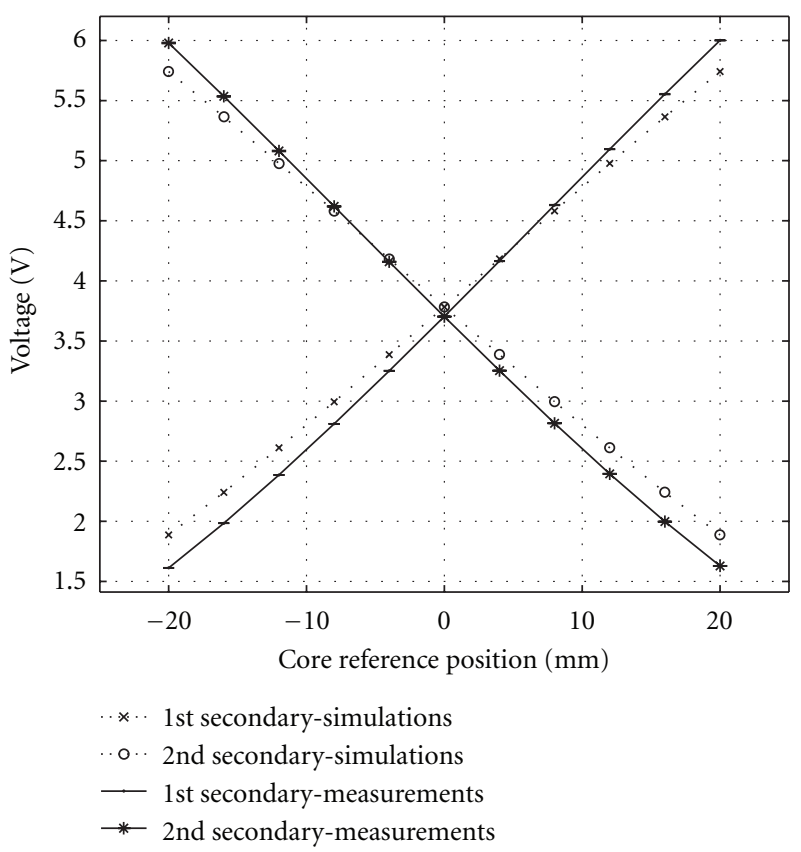

FIgURE 6: Simulations and measurements results regarding the LVDT secondary characteristic curve (current supply). The measurement data are depicted with the corresponding uncertainty.

3.2. Current Supply. The sensor has been fed with a current sine wave at $2 \mathrm{kHz}$ whose amplitude, $24.0 \mathrm{~mA}$, has been chosen in order to have the amplitude of the first harmonic of the primary voltage of about $3.5 \mathrm{~V}$ when the core is in null position. By doing so, the simulation results can directly be compared with the ones obtained with voltage supply.

The characteristic curve in the current supply case is displayed in Figure 6 (dotted line) for core positions going from $-20 \mathrm{~mm}$ to $+20 \mathrm{~mm}$.

Again, a dual behavior of the two curves in terms of trend and the symmetry around the null position can be noticed. In this case, the secondary transformation ratio of the sensor goes from 0.54 (minimum core coupling) to 1.66 (maximum core coupling).

Figure 7 (dotted line) shows the ratiometric curve with respect to the core position in current supply. It is very similar to the corresponding curve in voltage supply: the trend is monotonic (due to the LVDT working principle) and the ratiometric values range from about -0.5 to +0.5 , with a zero value in null position. Thus, the curve is once again symmetric with respect to the null position. In addition, the nonlinearity error $(0.7 \%$ on the full range, $0.12 \%$ in the range going from $-10 \mathrm{~mm}$ to $+10 \mathrm{~mm}$ ) is roughly the same which has been observed with voltage supply.

The results regarding the position drift are depicted in Figure 9 (dotted line). The shape and the magnitude depicted in that graph recalls the corresponding behavior in voltage supply; here, the position drift ranges from +72 micrometers to -72 micrometers, with a zero value in null position and higher values for positions which are farther from the center. 


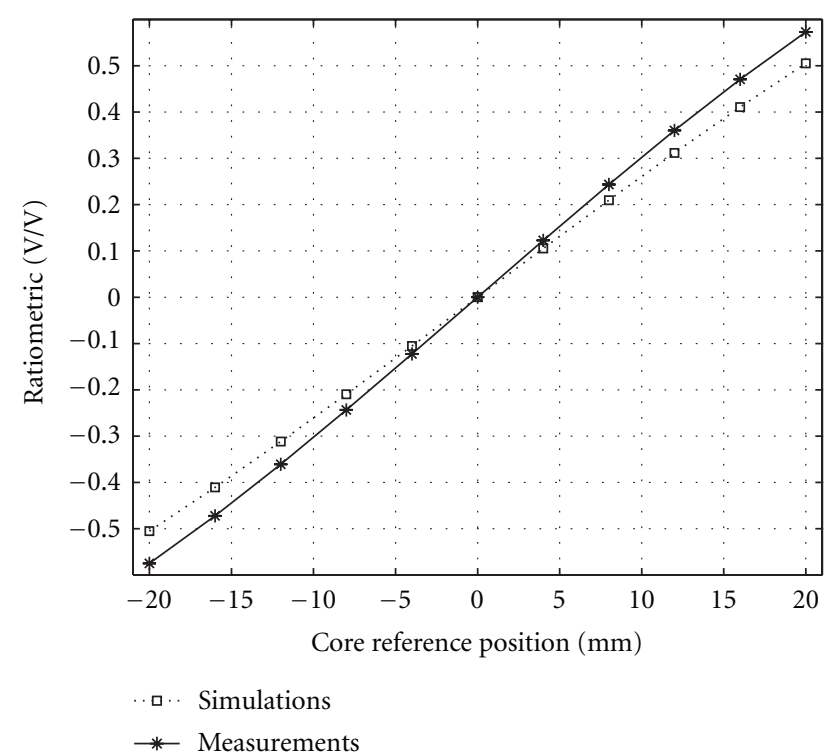

FIGURE 7: Simulations and measurements results regarding the ratiometric (current supply). The measurement data are depicted with the corresponding uncertainty.

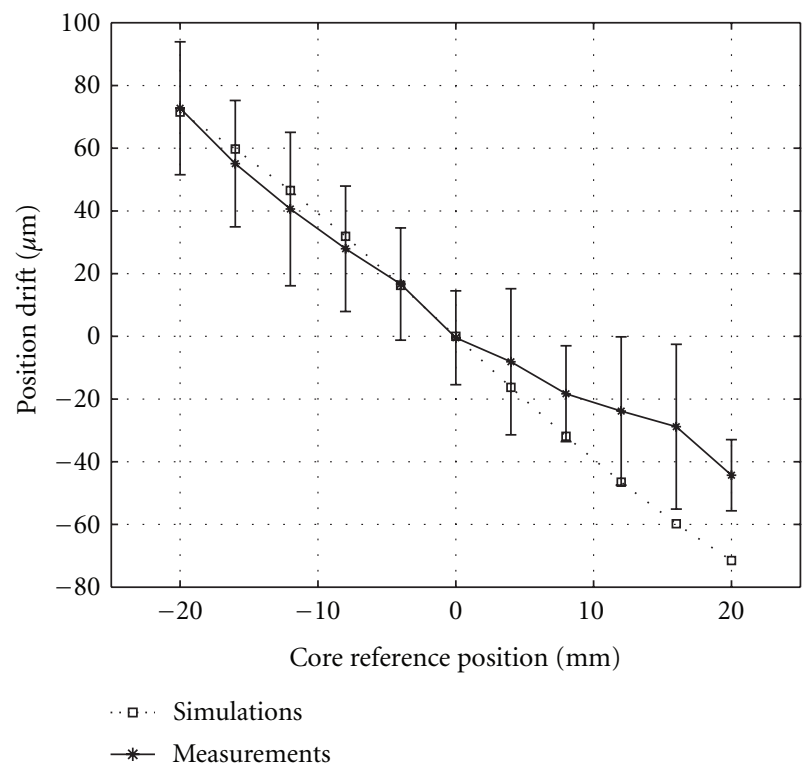

FIGURE 8: Simulations and measurements results regarding the position drift (voltage supply). The measurement data are depicted with the corresponding uncertainty.

\section{Experimental Validation}

4.1. Prototype Realization. The simulation work constituted the starting point for an LVDT prototype manufacturing. The dimensions, materials, geometry, and coils specifications reflect the simulation criteria. As already said in the last paragraph, all magnetic materials have been through an annealing process.

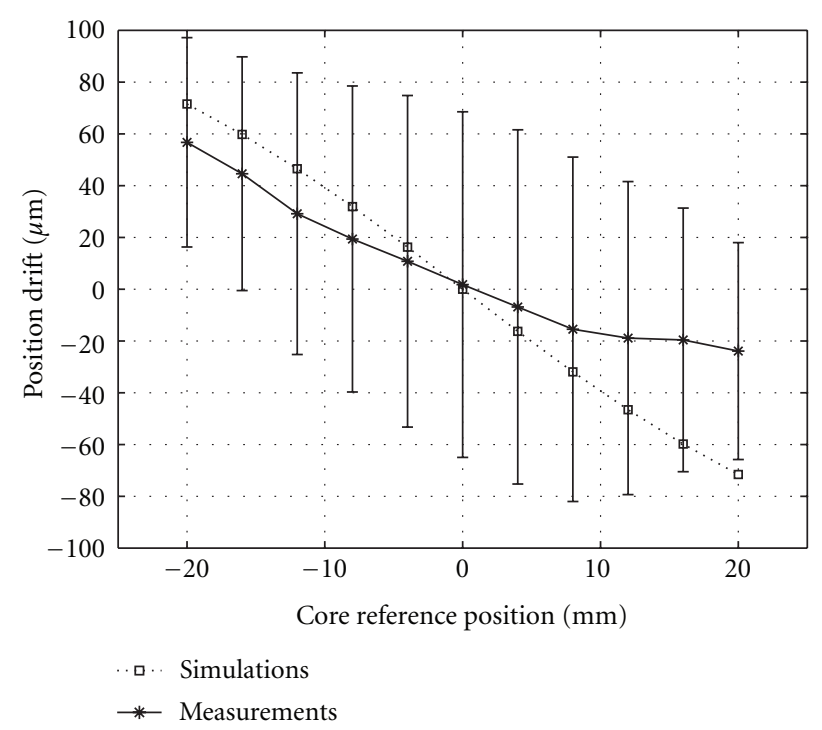

FIGURE 9: Simulations and measurements results regarding the position drift (current supply). The measurement data are depicted with the corresponding uncertainty.

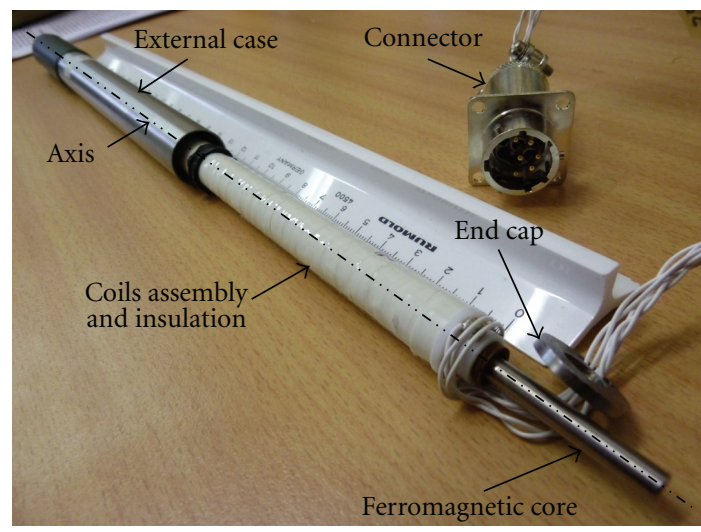

FIGURE 10: Expanded view of the custom prototype used to validate the FEM model. Dimensions scale is in centimeters.

The primary coil has been wound on 2 layers with a wire diameter of $0.28 \mathrm{~mm}$ (in order to allow possible measurements even with high currents) whereas the secondaries are single layer coils with a wire diameter of $0.06 \mathrm{~mm}$, since they are supposed to be connected to high impedances, typical of DAQs. An expanded view of the manufactured prototype, with highlighted information, is displayed in Figure 10.

4.2. Experimental Validation Procedure. A complete automatic test bench, whose details can be found in [11], has been used for the LVDT prototype metrological characterization, in both standard and magnetic interference conditions. In addition, the measurements have been performed in current and voltage supply. As for the FEM analysis, the core position range goes from $-20 \mathrm{~mm}$ to $20 \mathrm{~mm}$, but considerations have been done also for reduced ranges. In particular, the measurements at different positions are preceded by 
an iterative research of the null point of the sensor (electric zero research) [11].

The external longitudinal magnetic field is generated by means of an external calibrated solenoid, fed by a DC current so as to have the desired amplitude and a uniform distribution for the field along the solenoid length. In addition, a demagnetization procedure has been foreseen in the measurement process [11], in order to demagnetize the magnetic circuit of the sensor and keep as fixed as possible the static working point of the equivalent $\mathrm{B}-\mathrm{H}$ curve during the different measurement steps.

The measurements in absence of external magnetic field constitute the calibration of the prototype, yielding to its calibration curve. In presence of external field, the position is measured by using this curve, and the position drift is computed as the difference of the position measured in presence of external field and that measured in absence of it. As for the simulations, the position drift analysis is meant to be a relative study whose function is to point out the effect of the external magnetic interference.

In all experimental results, the related expanded measurement uncertainty is depicted too. This has been calculated on 30 repeated measurements, supposing the values spread as a Gaussian distribution in the measurement interval. A Chi-square test on the repeated electric zero research results confirmed that the null point of the LVDT prototype is spread as a Gaussian distribution with a standard deviation of 6 micrometers. The uncertainty of the measurements at different positions is, therefore, dominated by this component, since the other sources of uncertainty coming from the test bench give much smaller values [11]. The resulting expanded measurement uncertainty has been computed using a coverage factor 2 .

The uncertainty on the position drift has been calculated as follows:

$$
u_{\mathrm{drift}}=\sqrt{u_{p 1}^{2}+u_{p 0}^{2}}
$$

where $u_{p 0}$ and $u_{p 1}$ are the uncertainties on the measured position in absence and in presence of the external field, respectively. The difference between the values of such uncertainties in the different cases will be analyzed in the following subsections.

4.3. Voltage Supply. The prototype characteristic curve is depicted in Figure 4 (continuous line). The measurements showed that the secondary transformation ratio of the sensor goes from 0.48 (against a value of 0.54 for the simulations) to 1.69 (against a value of 1.66 for the simulations).

Regarding the voltage amplitudes, the agreement between simulation and measurements results is good, since it is always greater than $85 \%$ whereas it increases to more than $95 \%$ when reducing to $(-10 \mathrm{~mm},+10 \mathrm{~mm})$ the core position range.

The results for the ratiometric are depicted in Figure 5 (continuous line). In this case, the ratiometric is a monotonic function of the position rising from about -0.58 (against a value of -0.5 for the simulations) to about 0.57 (against a value of 0.5 for the simulations). Measurements results show a more pronounced nonlinearity, especially for high core position ranges $(1.11 \%$ in $(-20 \mathrm{~mm}, 20 \mathrm{~mm}))$. The graph in Figure 5 allows noticing the influence of the ratiometric reading on the match between simulations and measurements. As a matter of fact, while the match regarding the secondary voltages is more than $85 \%$, the one regarding the ratiometric is $83 \%$. Furthermore, such effect does not act uniformly on all the positions.

Regarding the interference conditions, the related position drift is presented in Figure 8 (continuous line). Some remarks on this figure have to be pointed out briefly. First of all, the drift at $0 \mathrm{~mm}$ is zero in the simulations and in the measurements (mean value): this is due to the fact that when the core is in the center, the interference effect on the two secondary windings is the same, resulting in a nonperturbed ratiometric reading (i.e., the measured position is not affected and the position drift is null). In addition, it can be stated that the good agreement between simulations and measurements (the simulation values are almost always within the measurement uncertainty in Figure 8) gets even better when considering only negative positions. As a matter of fact, the agreement in this area is more than $90 \%$. For positive positions, even if the simulations values are still within the measurement uncertainty, the agreement decreases. In other words, the intrinsic symmetry of the position drift which simulations have showed (Figure 8, dotted line) is not fully reflected by the measurements values (Figure 8, continuous line). In fact, for both simulations and measurements, the drift is positive for negative positions, negative for positive positions, and null in $0 \mathrm{~mm}$, but in the measurements the drift goes from 71 micrometers (against a value of 72 micrometers for the simulations) to -44 micrometers (against a value of -72 for the simulations). Such discrepancies are due to slight (micrometer order) asymmetric wire assembly of the prototype. Anyway, the overall agreement between FEM analysis and experimental measurements regarding the position drift is good.

A remark on the measurement uncertainty has to be done. In Figure 8 (continuous line), it can be noticed that the measurement uncertainty is meanly around 20 micrometers. This value of uncertainty depends, of course, on the uncertainty of the position measured in absence of the magnetic field and that of the position measured in presence of it, as in (3). These values are nevertheless higher than the corresponding values found in [11]. This is explained by taking into account that this prototype exhibits a high voltage swing (as displayed in Figure 4 and noted in Table 2) and so a big slope of the voltage-position characteristic (with respect to the sensors on which the test bench has been characterized in [11]); therefore, the uncertainty on the voltages is higher (with respect to the ones found in [11]) and so is that on the measured position.

4.4. Current Supply. The measurements in current supply have been performed by using a sinusoidal feeding current whose peak amplitude is $23.0 \mathrm{~mA}$. This value ensures a first harmonic amplitude of the primary voltage of $3.5 \mathrm{~V}$ when the core is in null position. This current value is in agreement with the one coming from the simulations; thus, there 
TABLE 2: Summary of experimental results.

\begin{tabular}{|c|c|c|}
\hline Item & Voltage supply & Current supply \\
\hline Feeding signal amplitude & $3.5 \mathrm{~V}(3.5)$ & $23.0 \mathrm{~mA}(\mathbf{2 4 . 0})$ \\
\hline Transformation ratio & from $0.48(\mathbf{0 . 5 4})$ to $1.69(\mathbf{1 . 6 6})$ & from $0.48(\mathbf{0 . 5 4})$ to $1.70(\mathbf{1 . 6 6})$ \\
\hline Ratiometric range & from $-0.58(-\mathbf{0 . 5 0})$ to $0.57(\mathbf{0 . 5 0})$ & from $-0.57(-\mathbf{0 . 5})$ to $0.57(\mathbf{0 . 5})$ \\
\hline Voltage swing (V) & $4.30(3.90)$ & $4.35(3.90)$ \\
\hline Non linearity error (\%) & $\begin{array}{l}1.11(\mathbf{0 . 7}) \text { in }(-20 \mathrm{~mm}, 20 \mathrm{~mm}) \\
0.14(\mathbf{0 . 1 1}) \text { in }(-10 \mathrm{~mm}, 10 \mathrm{~mm})\end{array}$ & $\begin{array}{l}1.12(\mathbf{0 . 7}) \text { in }(-20 \mathrm{~mm}, 20 \mathrm{~mm}) \\
0.15(\mathbf{0 . 1 1}) \text { in }(-10 \mathrm{~mm}, 10 \mathrm{~mm})\end{array}$ \\
\hline Primary voltage repeatability $(\mathrm{mV})$ & $\begin{array}{c}0.8 \text { (no interference }) \\
2.5 \text { (with interference) }\end{array}$ & $\begin{array}{l}1.5 \text { (no interference) } \\
8 \text { (with interference) }\end{array}$ \\
\hline Secondary voltage repeatability $(\mathrm{mV})$ & $\begin{array}{c}1.6 \text { (no interference) } \\
2.9 \text { (with interference) }\end{array}$ & $\begin{array}{c}2.1 \text { (no interference) } \\
8.8 \text { (with interference) }\end{array}$ \\
\hline $\begin{array}{l}\text { Measured position uncertainty } \\
\text { (micrometers) }\end{array}$ & $\begin{array}{c}11 \text { (no interference) } \\
15 \text { (with interference) }\end{array}$ & $\begin{array}{c}13 \text { (no interference) } \\
52 \text { (with interference) }\end{array}$ \\
\hline Position drift values (micrometers) & from $71(72)$ to $-44(-72)$ & from $58(72)$ to $-25(-72)$ \\
\hline Position drift uncertainty (micrometers) & 19 & 55 \\
\hline $\begin{array}{l}\text { Agreement with simulations } \\
\text { (characteristic curve) }(\%)\end{array}$ & $\begin{array}{l}>85 \mathrm{in}(-20 \mathrm{~mm}, 20 \mathrm{~mm}) \\
>95 \mathrm{in}(-10 \mathrm{~mm}, 10 \mathrm{~mm})\end{array}$ & $\begin{array}{l}>86 \text { in }(-20 \mathrm{~mm}, 20 \mathrm{~mm}) \\
>92 \text { in }(-10 \mathrm{~mm}, 10 \mathrm{~mm})\end{array}$ \\
\hline $\begin{array}{l}\text { Agreement with simulations } \\
\text { (ratiometric) }(\%)\end{array}$ & $>83$ & $>84$ \\
\hline
\end{tabular}

is a good match between measurements and simulations, regarding the primary impedance.

Figure 6 (continuous line) shows the measured characteristic curve in current supply. The measured transformation ratios go from 0.48 (against a value of 0.54 for the simulations) to 1.70 (against a value of 1.66 for the simulations). The agreement regarding the amplitudes is good in this case too, since the characteristic curves exhibit an agreement of more than $86 \%$; such value increases to $94 \%$ when reducing to $(-10 \mathrm{~mm}, 10 \mathrm{~mm})$ the core position range.

The behavior of the ratiometric values with respect to the core position is shown in Figure 7 (continuous line). The ratiometric ranges from -0.57 (against a value of 0.5 for the simulations) to 0.57 (against a value of 0.5 for the simulations). In this case, the non linearity (calculated as in (2)) is $1.12 \%$ in $(-20 \mathrm{~mm}, 20 \mathrm{~mm})$. As already observed for the voltage supply case, the ratiometric reading nonuniformly affects the match between simulations and measurements, since the agreement is here equal to $84 \%$ in the worst case.

Concerning the interference case, the results regarding the position drift are depicted in Figure 9 (continuous line). As for the simulations, the position drift is a monotonic function of the core position and its value for null position is null on turn. In addition, the agreement with FEM results is good, especially for negative positions (as already observed with voltage supply), since the simulation values are almost always within the measurement uncertainty.

Furthermore, a remark on this agreement in current supply has to be done. The secondary voltage of an LVDT can be expressed as [12]

$$
V_{i} \propto I_{p} \cdot M_{i p}(p) \cdot m\left(H_{\mathrm{ext}}\right)
$$

where $I_{p}$ is the amplitude of the primary current, $M_{i p}$ is the mutual inductance between the ith secondary and the primary, $m$ is an adimensional factor which takes into account the dependence of such mutual inductance on the external magnetic field. When an external field is applied, the voltage variation is

$$
\Delta V_{i} \propto I_{p} \cdot M_{i p}(p) \cdot \Delta m
$$

Consequently, the dependence of the position drift on the position will be connected with that of the mutual inductance (being the only factor which is a function of the position in (5)). Therefore, the agreement on the slope of the drift-position curve (Figure 9) has to be expected similar to the agreement on the slope already observed for the voltageposition curve in Figure 6, which is $84 \%$. This is actually the case (for negative positions, as already stated). According to the model presented in [12], this is valid for current supply.

Regarding the measurement uncertainty on the position drift, in this case it is higher than the correspondent with voltage supply. Actually, with current supply the uncertainty on the primary voltage is higher (the primary voltage is not fixed by the generator), as reported in Table 2, leading to a higher uncertainty on the secondary voltages and finally on the position drift.

For a complete overview of the results in different supply cases and the comparison with simulations, see Table 2. In this table, the numerical results regarding the measurements in the different supply cases are summarized and compared with the corresponding values obtained with the FEM simulations, reported in brackets and expresses in the same measurement units.

\section{Conclusions and Outlook}

The finite element analysis is used in this work to conceive a FEM model of LVDT for the study of the effect of magnetic interference on the sensor. The model is presented together 
with simulation results of the LVDT characteristic curve and the effect of a $1 \mathrm{mT}$ external flux density on the position reading. A custom LVDT prototype has been manufactured in order to validate the model, by means of an automated measurement test bench [18]. A good agreement with respect to simulations has been observed for the LVDT characteristic curves and for the position drift due to the external magnetic field.

The scientific target of the proposed FEM model and the simulation procedure described in standard working conditions and with external interference is to make available a tool both for the study of the physical phenomenon and for the design of future LVDTs where the sensitivity to external field can be correctly taken into account from the very preliminary phase.

The future design of an LVDT with less sensitivity to external magnetic fields could indeed profit from considerations and results based on the FEM model here presented: for example, the simulation data can affect the improvement of the performances of an LVDT by giving rapid design feedbacks regarding the choice of a certain material for the core or for an external magnetic shield, or allowing a sensitivity study with different coiling specifications, magnetic field intensity and orientation, and so forth. Furthermore, the proposed model and its future expansion can significantly support the study (analytical or FEM aided) of a more complex and general case of magnetic interference on linear variable differential transformers (e.g., considering nonuniform and slowly varying interfering magnetic fields).

\section{Acknowledgments}

The authors would like to thank engineer Antonio Pierno and Dr. Daho Taghezout (applied Magnetics) for the support on measurements and simulations respectively. They would also like to thank Professor Vittorio Giorgio Vaccaro, Professor Felice Cennamo and Professor Yves Perriard for their useful discussions and suggestions.

\section{References}

[1] D. S. Nyce, “The LVDT," in Linear Position Sensors, vol. 98, pp. 94-97, Wiley-Interscience, Hoboken, NJ, USA, 2004.

[2] A. Masi, A. Brielmann, R. Losito, and M. Martino, "LVDT conditioning on the LHC collimators," IEEE Transactions on Nuclear Science, vol. 55, no. 1, pp. 67-75, 2008.

[3] R. S. Weissbach, D. R. Loker, and R. M. Ford, "Test and comparison of LVDT signal conditioner performance," in Proceedings of the IEEE Instrumentation and Measurement Technology (IMTC '00), pp. 1143-1146, May 2000.

[4] D. Crescini, A. Flammini, D. Marioli, and A. Taroni, "Application of an FFT-based algorithm to signal processing of LVDT position sensors," IEEE Transactions on Instrumentation and Measurement, vol. 47, no. 5, pp. 1119-1123, 1998.

[5] K. Ara, "A differential transformer with temperature-and excitation-lndependent output," IEEE Transactions on Instrumentation and Measurement, vol. 21, no. 3, pp. 249-255, 1972.

[6] A. Masi and R. Losito, "LHC collimators low level control system," IEEE Transactions on Nuclear Science, vol. 55, no. 1, pp. 333-340, 2008.
[7] R. Bozorth, Ferromagnetism, D. Van Nostrand Company, New York, NY, USA, 3rd edition, 1951.

[8] K. Hoselitz, "Magnetically soft materials," in Ferromagnetic Properties of Metals and Alloys, pp. 238-239, Clarendon Press, Oxford University, Oxford, UK, 1952.

[9] M. Martino, A. Danisi, R. Losito, A. Masi, and G. Spiezia, "Design of a Linear Variable Differential Transformer with High Rejection to External Interfering Magnetic Field," IEEE Transactions on Magnetics, vol. 46, no. 2, Article ID 5393179, pp. 674-677, 2010.

[10] A. Danisi, Simulation of DC interfering magnetic field effects on the LHC collimators' LVDT positioning sensors, M.S. thesis, Federico II, Naples, Italy, 2009, https://edms.cern.ch/,EDMS Id 1016998.

[11] G. Spiezia, R. Losito, M. Martino, A. Masi, and A. Pierno, "Automatic test bench for measurement of magnetic interference on LVDTs," IEEE Transactions on Instrumentation and Measurement, vol. 60, no. 5, pp. 1802-1810, 2011.

[12] M. Martino, G. Golluccio, R. Losito and A. Masi, "An analytical model of the effect of external DC magnetic fields on the AC voltages of an LVDT," in Proceedings of the IEEE Instrumentation and Measurement Technology Conference, Austin, Tx, USA, May 2010.

[13] J. K. Sykulski, E. Sykulska, and S. T. Hughes, "Application of finite element modelling in LVDT design," COMPEL, vol. 11, no. 1, pp. 73-76, 1992.

[14] M. Repetto and J. Simkin, "Engineering analysis for design optimisation of differential transformers," Computer-Aided Engineering Journal, vol. 5, no. 2, pp. 51-53, 1988.

[15] Y. Kano, S. Hasebe, and H. Miyaji, "New linear variable differential transformer with square coils," IEEE Transactions on Magnetics, vol. 26, no. 5, pp. 2020-2022, 1990.

[16] S. C. Saxena and S. B. Lal Seksena, "Self-compensated smart LVDT transducer," IEEE Transactions on Instrumentation and Measurement, vol. 38, no. 3, pp. 748-753, 1989.

[17] “Flux ${ }^{\circledR} 9$ 2D/3D Applications User’s Guide," CEDRAT, Meylan, France, 2006.

[18] M. Krizek and P. Neittaanmaki, "Finite elements," in Mathematical and Numerical Modelling in Electrical Engineering, pp. 76-77, Kluwer Academic, Dordrecht, The Netherlands, 1996.

[19] I. K. Kang, H. H. Scholefield, and A. P. Martin, "Some concepts concerning the new high-permeability 50-50 nickel-iron alloys," Journal of Applied Physics, vol. 38, no. 3, pp. 1178-1183, 1967. 

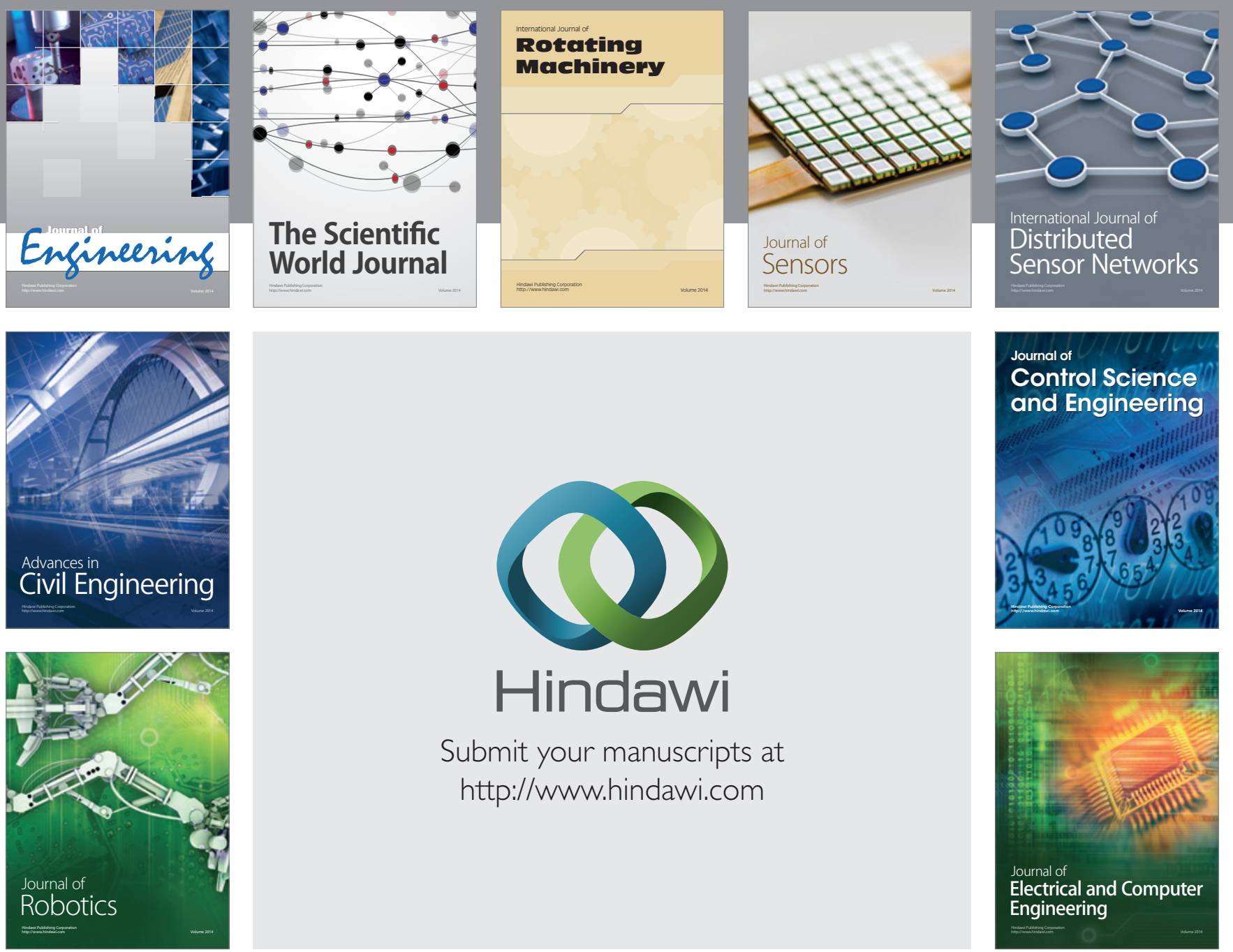

Submit your manuscripts at

http://www.hindawi.com
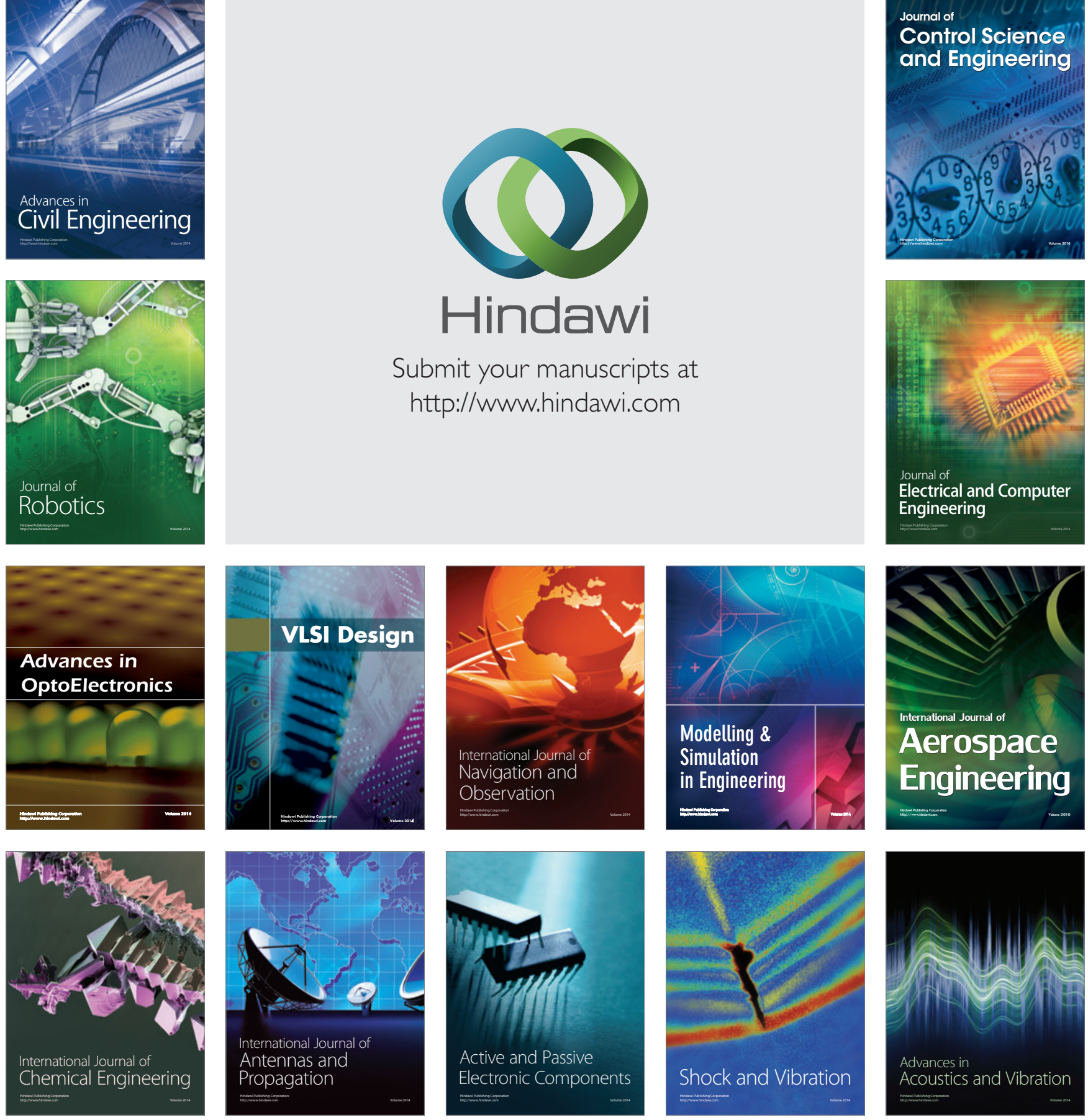